\title{
COMPUTER AIDED PROCESSING OF CERVICAL SPINE RADIOGRAPHS
}

Petra Aradi ${ }^{1}$, László Danka ${ }^{2}$, György Lipovszki ${ }^{1}$, Ildikó Németh ${ }^{3}$

${ }^{1}$ Budapest University of Technology and Economics, Faculty of Mechanical Engineering, Department of Mechatronics, Optics and Engineering Informatics

${ }^{2}$ Policlinic of Hospitaller Brothers of St. John of God, Department of Orthopedics

${ }^{3}$ Dr. Rose Kft.

aradi.petra.bme@gmail.com

\begin{abstract}
Quite a number of medical specialties require distance and angle measurements on x-ray images. Drafting and measurement on traditional $\mathrm{x}$-ray films can be done with marker pens, rulers and protractor, but this method is time consuming and often inaccurate. Authors present a computer aided drafting and measurement method for both digitized and digital x-rays.

Evaluation of cervical spine $\mathrm{x}$-rays is especially important for patients suffering from rheumatoid arthritis. Our computer program helps users to mark necessary points and draw lines on $\mathrm{x}$-rays interactively on the computer screen, then the program calculates relevant angles and distances based on literature data.
\end{abstract}

Manual drafting on traditional x-ray films is time consuming, and it is usually impossible or at least difficult to repeat. When one wants to try drafting more than once, previous lines have to be erased from the film, or a separate transparent film can be used. Reading distance and angle values requires a ruler and a protractor. The tip size of the marker pen and the precision of ruler, protractor positioning can result in measurement errors of multiple millimeters and degrees.

Contrarily, computer based drafting allows arbitrary number of users arbitrary number of attempts to define necessary points on x-rays, and then predefined algorithms calculate the results.

Computer aided drafting can be performed by many persons, a number of times. Accuracy can be improved by using image processing capabilities such as zooming, contrast correction, etc. Results can be superimposed on x-ray images, making follow-up easier.

Compared to ,traditional” drafting, the computer-aided method's repeatability and reliability is a great advantage. It is easier to learn computer-based drafting, especially when a large number of points have to be marked and complicated measurements are involved. With an appropriate drafting and evaluation protocol, processing of x-rays provides added diagnostic value, e.g. further examinations can be ordered based on the results, scheduling of follow-ups can be optimized.

Keywords: functional radiograph, rheumatoid arthritis, digital processing, distances and angles.

\section{Introduction}

As stated in the reference ${ }^{1}$ joint destruction in rheumatoid arthritis patients' cervical spine may lead to progressive vertebral instability. The consequences of this condition can lead to sudden death under certain conditions, e.g. during anaesthesia. Physical symptoms are not always in 
correlation with joint destruction, meaning symptomless patients might very well be endangered. Cervical spine deformities can be visualized on radiographs. Five standard $\mathrm{x}$-ray settings are used: conventional bidirectional (anteroposterior and lateral), transoral dens and functional (performed in flexion and extension) lateral images are taken. This study discusses the importance of such radiographic examinations, both as preoperative evaluation and as follow-up examination. Based on the results of such examinations, patients can be directed to other imaging tests, such as $\mathrm{x}$-rays in other directions, CT, MRI, to decide on eventual operative fixation.

In order to uniformly evaluate this radiograph series, various methods are to be found in international literature. Common in these methods is the use of lines directed through certain anatomical points. Distances and angles are calculated from these lines, providing the possibility of diagnostic classification. Traditional drafting methods involve the use of marker pens, rulers, a protractor and a traditional x-ray film. Manual drafting on traditional x-ray films is time consuming on the one hand, and it is usually impossible or at least difficult to repeat on the other hand. Difficulties come up if drafting has to be performed more than once, or the original radiograph without pen lines has to be kept as well. Further errors can occur from too wide tip size of the marker pen, precision of measurement devices, and reading errors. Another problem arises from the use of digital x-rays, when the image is normally not printed, so that drafting can be performed.

Physicians evaluating such series of radiographs require thorough training in this relatively time (and resource) consuming drafting process and in determining required distances and angles.

To get around these difficulties, a computer program is developed. The program can use digital $\mathrm{x}$-ray images natively, while traditional x-ray films have to be digitized. Another advantage is the systematic onscreen "drafting", where the evaluation protocol is to be followed, minimizing the chances of error from incorrectly marked points and lines. A further asset is that the use of the program requires less experience, than "traditional" drafting. Other advantages of computer processing include automated calculation of distances and angles, as well as the opportunity of evaluation by multiple users, more than once, and digital storage of images, drafted lines and results.

\section{Methods and materials}

\section{X-ray protocol}

The method ${ }^{1}$ developed at Policlinic of Hospitaller Brothers of St. John of God is systematically covers screening, measuring and grading of cervical subluxations and instability. The protocol specifies how x-rays have to be made (Table 1 and Figure 1). ${ }^{1}$ 


\begin{tabular}{|c|l|c|}
\hline$\#$ & Description & Sample \\
\hline 1 & $\begin{array}{l}\text { Anteroposterior axis, } 100 \mathrm{~cm} \text { focus-film distance, } 10^{\circ} \text { caudocranial tilt, centered to } \\
\text { C3 }\end{array}$ & Figure 1a \\
\hline 2 & $\begin{array}{l}\text { Lateral direction, } 150 \mathrm{~cm} \text { focus-film distance, horizontal primary beam, centered to } \\
\text { C2 }\end{array}$ & Figure 1b \\
\hline 3 & $\begin{array}{l}\text { Lateral direction, flexion with chin pressed down, } 150 \mathrm{~cm} \text { focus-film distance, } \\
\text { horizontal primary beam, centered to C3 }\end{array}$ & Figure 1c \\
\hline 4 & $\begin{array}{l}\text { Lateral direction, extension with head tilted back, } 150 \mathrm{~cm} \text { focus-film distance, } \\
\text { horizontal primary beam, centered to C3 }\end{array}$ & Figure 1d \\
\hline 5 & $\begin{array}{l}\text { Anteroposterior axis, transoral dens image, } 50 \mathrm{~cm} \text { focus-film distance, } \\
\text { perpendicular primary beam, centered to dens epistrophei }\end{array}$ & Figure 1e \\
\hline
\end{tabular}

Table 1. X-ray protocol for rheumatoid arthritis ${ }^{1}$
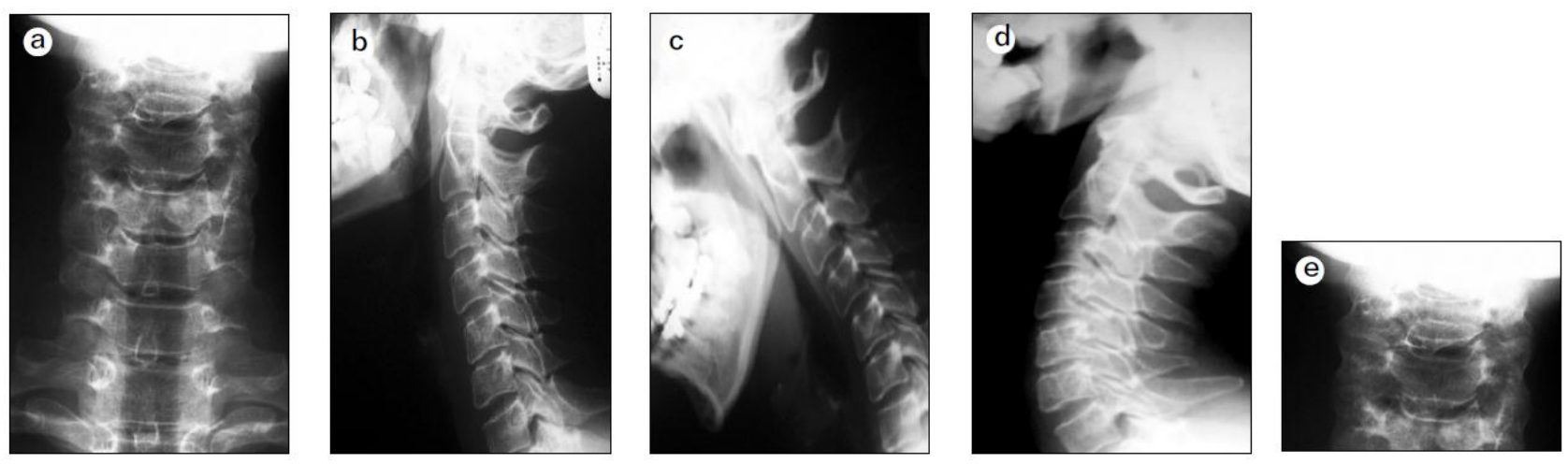

Figure 1. Sample images ${ }^{1}$ prepared according to $\mathrm{x}$-ray protocol for patients with rheumatoid arthritis

a: Anteroposterior, b: Lateral, c: Functional lateral (flexion), d: Functional lateral (extension), e: Transoral anteroposterior dens

\section{Drafting protocol}

Drafting protocol, e.g. specific points and lines, developed at Policlinic of Hospitaller Brothers of St. John of God is composed of a number of evaluation methods from international literature.

There are two types of x-rays, anteroposterior (normal and transoral dens), and lateral (neutral, fully flexed and extended). The points, lines and distances needed in evaluation of transoral dens images are shown in Table 2 and Figure 2.

\begin{tabular}{|c|l|}
\hline Object id & Description \\
\hline 1 & intervestibular line \\
\hline 2 & bimastoid line \\
\hline 3 & transspinous-transdental line going through the central line of dens \\
\hline 4 & distance from dens to lateral mass of atlas on both sides \\
\hline
\end{tabular}

Table 2. Objects to be marked on anteroposterior transoral dens radiograph ${ }^{1}$ 


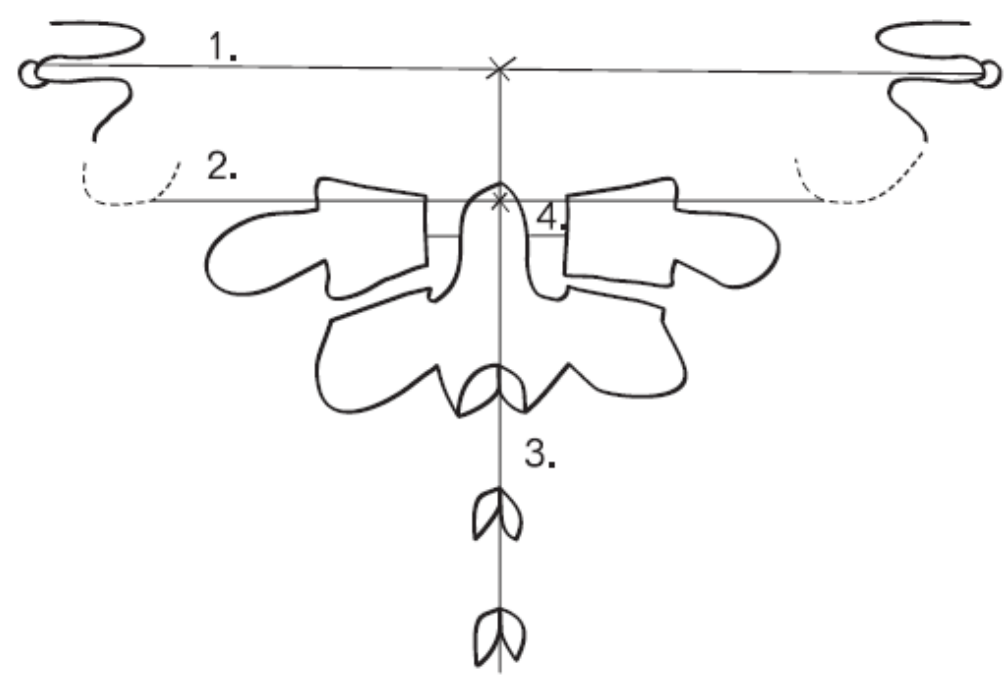

Figure 2. Lines to be drawn on anteroposterior transoral dens radiograph ${ }^{1}$

Lateral radiographs are evaluated with the use of points, lines and angles specified in the above mentioned protocol, and are presented in Table 3 and Figure 3.

\begin{tabular}{|c|l|}
\hline Object id & Description \\
\hline 1 & $\begin{array}{l}\text { atlantodental interval (ADI) is measured between the line drawn over the dorsal } \\
\text { contour of C1's anterior tubercle and the ventral surface of dens }\end{array}$ \\
\hline 2 & posterior atlantodental interval (PADI) is the retrodental space \\
\hline $3 a$ & lower atlas line in Sakaguchi-Kauppi method \\
\hline $3 b$ & tangential line of upper arc of C2's pedicle in Sakaguchi-Kauppi method \\
\hline 4 & $\begin{array}{l}\text { subaxial central canal (sAC) is the shortest distance between dorsal vertebral line (8) } \\
\text { and spinolaminar line (7) on a given level }\end{array}$ \\
\hline 5 & $\begin{array}{l}\text { line in McGregor method connects dorsal endpoint of os palatum with outer contour of } \\
\text { occiput, and the relative position of the dens' tip is compared to it }\end{array}$ \\
\hline 6 & $\begin{array}{l}\text { in Wackenheim method a line is drawn down the posterior surface of the clivus and its } \\
\text { position is compared to the posterior aspect of dens }\end{array}$ \\
\hline 7 & $\begin{array}{l}\text { spinolaminar line is an arc connecting the inner contours of spinous processes from the } \\
\text { posterior margin of foramen magnum }\end{array}$ \\
\hline 8 & posterior vertebral line is an arc above the dorsal contours of vertebral bodies \\
\hline 9 & $\begin{array}{l}\text { processus spinosus relation is the distance between two neighboring spinous processes } \\
\text { (lower arch of the upper one and the upper arch of the spinous process below) }\end{array}$ \\
\hline 10 & angle of lines drawn at the lower surfaces of two neighboring vertebrae \\
\hline
\end{tabular}

Table 3. Objects to be marked on lateral radiographs (neutral, flexed, extended) ${ }^{1}$ 


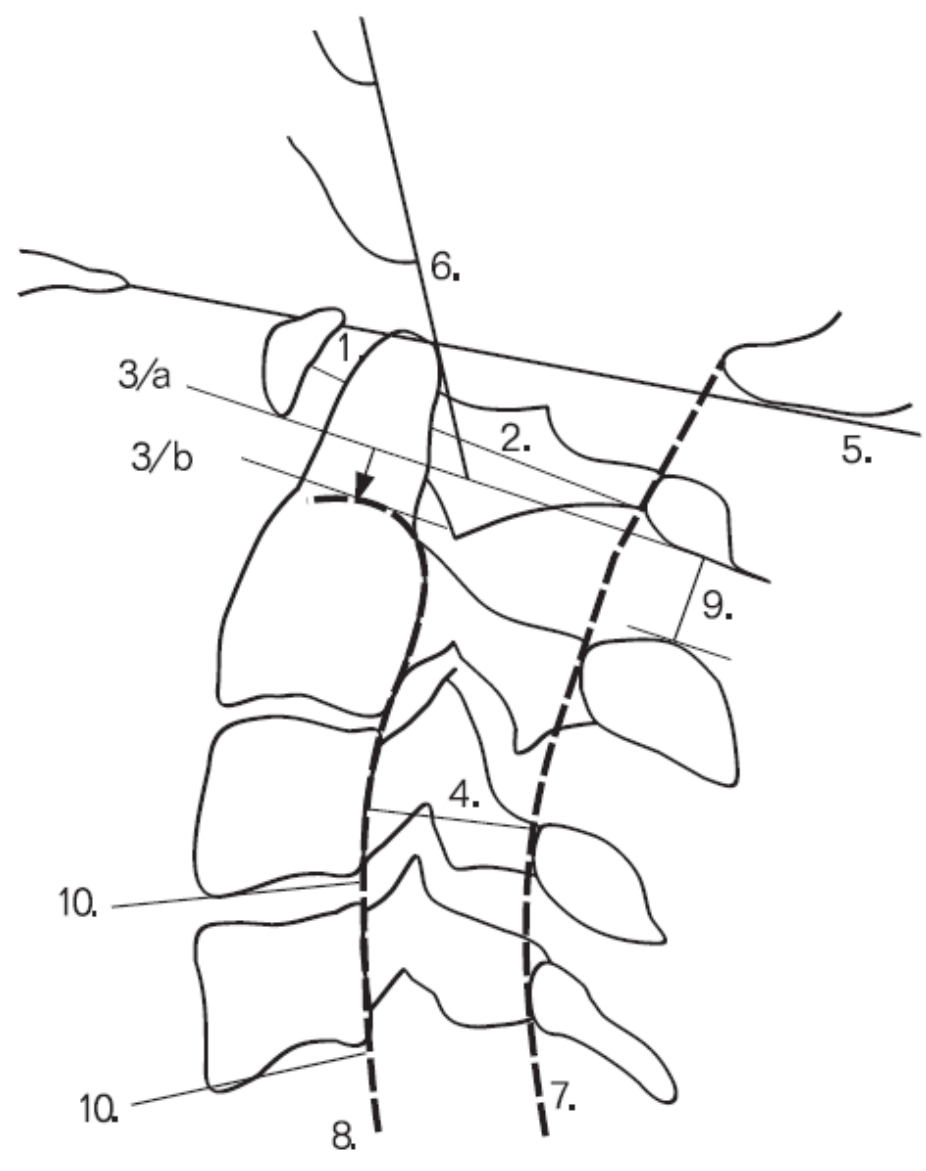

Figure 3. Objects to be marked on lateral radiographs ${ }^{1}$

\section{Results}

\section{Drafting and evaluation program}

2009 versions of National Instruments LabVIEW $^{2}$ and NI Vision ${ }^{3}$ were used to implement computer based drafting and for calculating relevant distances and angles.

The program has a main window (Figure 3), to control execution. Each function (selecting radiographs, evaluating various images and finally displaying results) can be accessed with a button. At the moment everything (button labels, instructions, etc.) are bilingual.

Functions have to be performed in a certain order, so it is not possible to press the five drafting buttons, until the first task, namely selecting radiographs has not been completed. The completion of each task is indicated by a round LED next to the button (e.g. the first task is completed in Figure 3). Similarly, it is not possible to press the Results button, until at least one evaluation has not been completed. When the user presses a button, a new window opens, and after finishing the selected task it is closed, and the user is returned to the main window. The 
Stop button in the lower right corner of the main program window allows stopping the program; however without performing the saving of results, each and every drawn line and calculated value will be lost. To avoid accidental data loss, pressing Stop pops up a confirmation dialog.

Traditional x-ray films have to be digitized (into JPG format), DICOM digital images has to be converted to JPG. When the program asks the user for the five images, user has to select the one that is specified in the title row of each File Open dialog box (Figure 4).

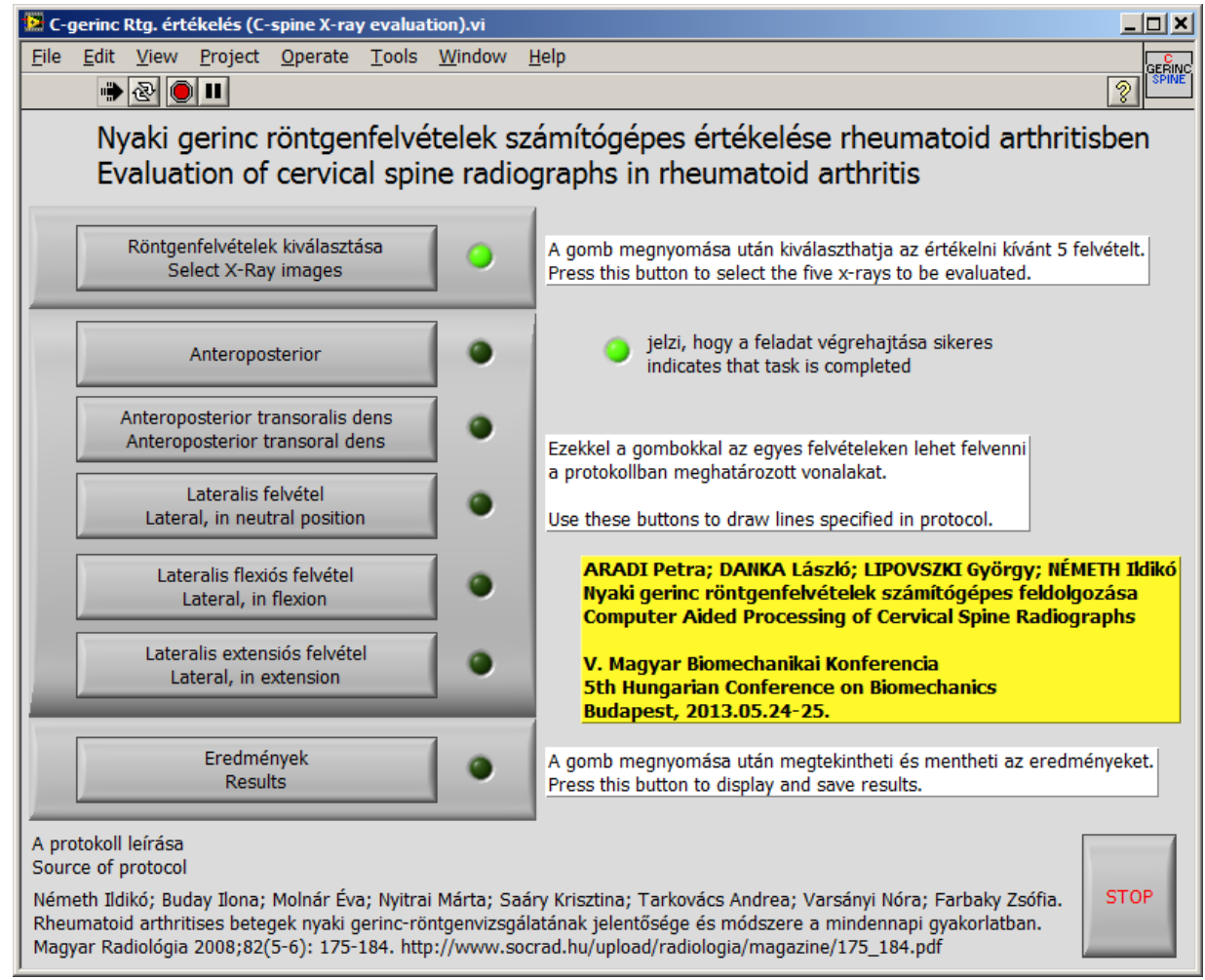

Figure 3. Main program window

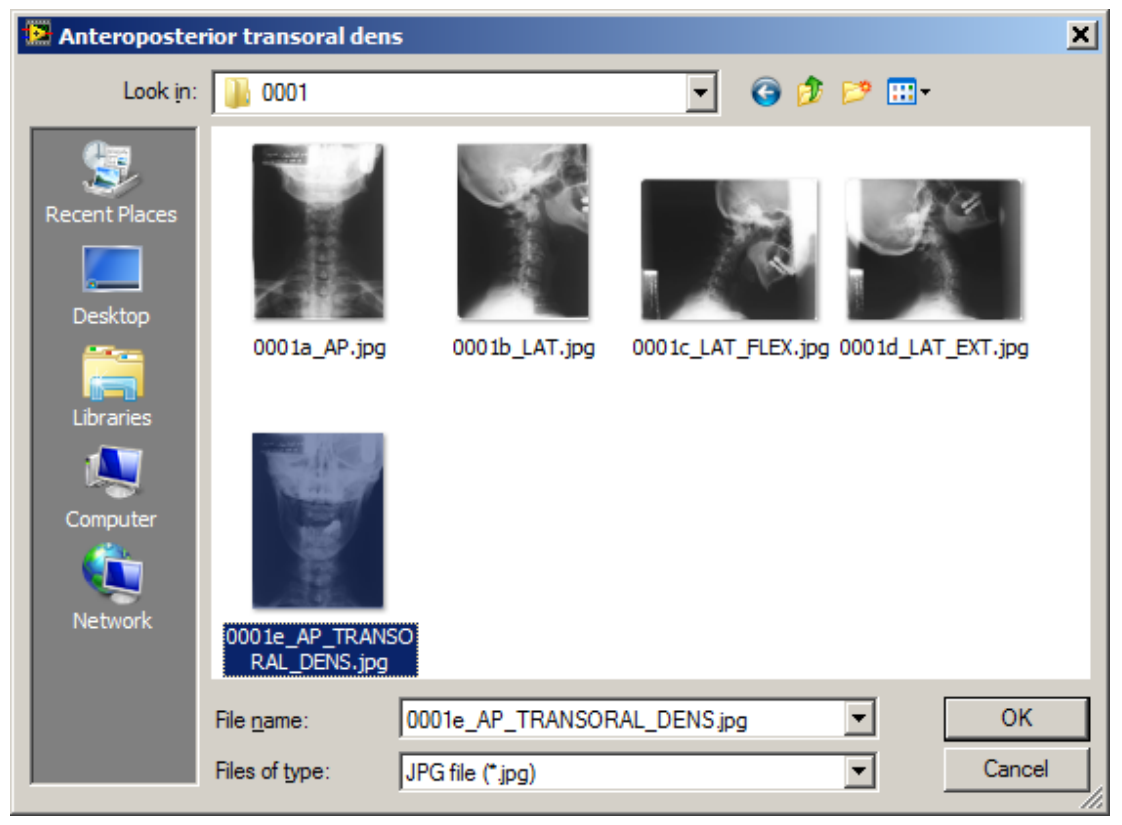

Figure 4. Selecting and opening JPG format radiographs one by one 
The user interface in each of the drafting tasks shows the graphics (Figures 2,3) and bilingual text guides (Tables 2,3) from the protocol as a help for users. In Figure 5 evaluation steps for the anteroposterior transoral dens radiograph are presented, showing that step 3 is already completed. In the small image in the lower left part of the window the already drawn transspinous-transdental line is visible. When the user presses one of the numbered buttons, a new window opens up with the image sized to the maximal vertical screen resolution, in order to make drawing not too difficult and as precise as possible. Until this image window is open, the line can be modified by its endpoints or moved as a unit. Each numbered drawing step can be redone as many times, as it suits the user. Stop button can be pressed anytime, without even performing any drawing; however it has no use, because it does not produce any lines and consequently nothing can be calculated.

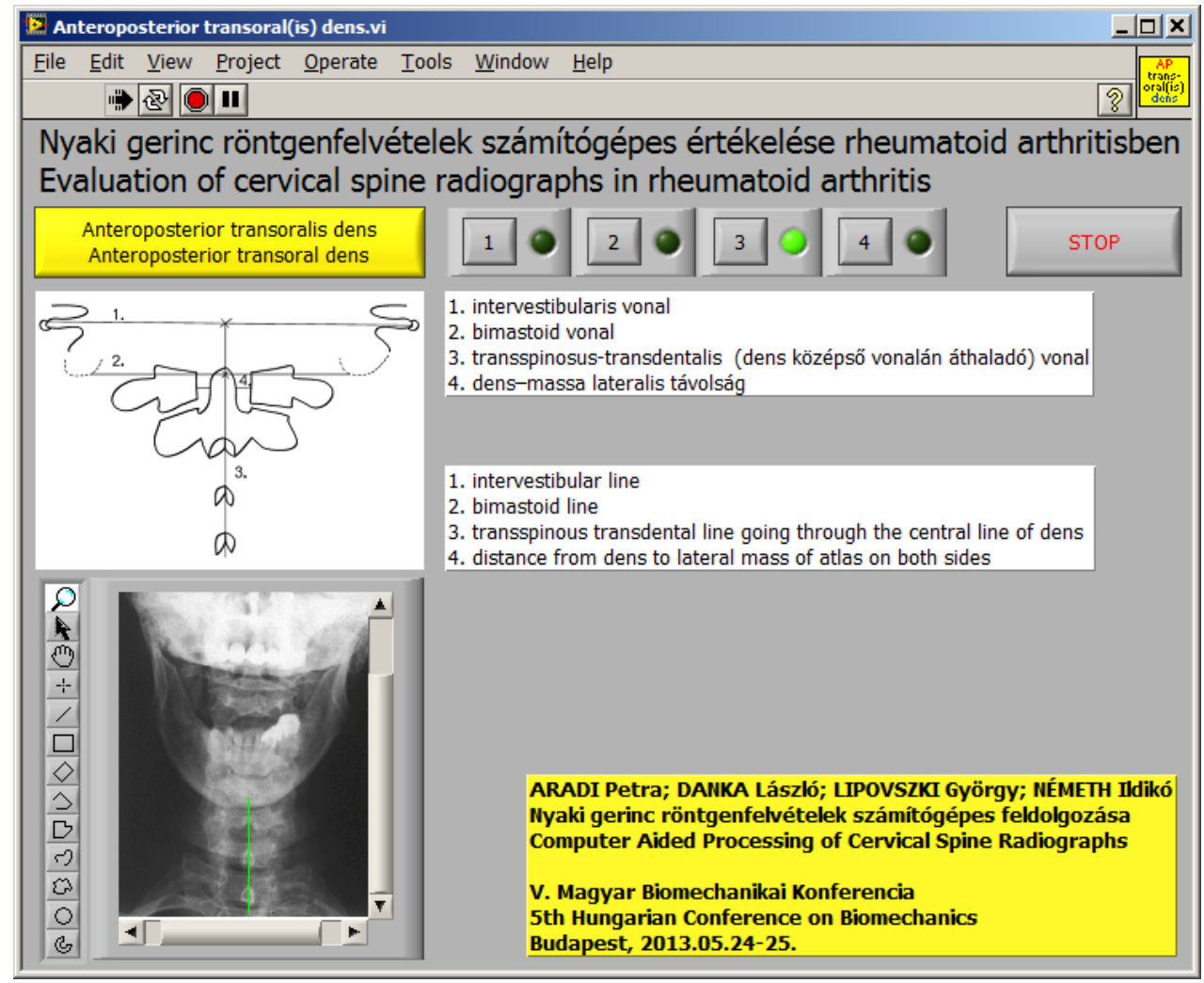

Figure 5. Drafting on anteroposterior transoral dens radiograph with step-by-step instructions

Windows similar to the one in Figure 5 are available for all the other images. Because of the interdependency of elements drawn on lateral images (as shown in Table 3), e.g. step 4 requires lines 7 and 8, user have to draw these two lines first, to enable button 4 .

When drafting tasks are completed and Results button is pressed, first patient's data can be set, as well as the evaluating person and other relevant data. The program then performs the necessary calculations to provide results needed to determine patient's status. Pathologic values and the 
recommended diagnostic procedures from the referenced artice1 are included in the program, so that it can mark them and classify the type and degree of atlantoaxial subluxation accordingly.

For the time being, the possibility of drawing other widely used auxiliary lines for methods, which are not part of the protocol, such as Ranawat, or Redlung-Johnell-method, a button is set in the Results section. By pressing this button similar drafting windows appear, and results can be obtained, too.

\section{Conclusion}

The program is still under extensive testing and development, according to users' needs; however one can immediately see the positive difference it brings, compared to traditional manual drafting. Among the advantages one have to mention that drafting and evaluation can be repeated, results as numbers, text and lines superimposed on radiographs can be stored.

Further development might include some kind of automation in recognizing points to be used to draw the lines, true multilingual user interface, etc.

\section{REFERENCES}

1. Bíró I, Csizmadia BM, Katona G. Determination of instantaneous axis of rotation of tibia and its role in the kinematical investigation of human knee joint. In: L. Borbás editor. Proceedings of the Third Hungarian Conference on Biomechanics; 2008. July 4-5. Budapest; 2008:57-62.

2. Fekete G, De Baets P, Wahab MA, Csizmadia BM, Katona G, Vanegas-Useche LV, Solanilla JA. Sliding-rolling ratio during deep squat with regard to different knee prostheses. Acta Polytechnica Hungarica 2012; 9(5): 5-24.

3. Szakál Z. Mérőberendezés térdízület mozgásvizsgálatához. GÉP 2006; LVII(1): 37-40.

The authors would like to thank National Development Agency (NDA) of Hungarian Government for its support since this study has been carried out commonly as part of project GERINCO2 TECH_08-A1/2-2008-0121. 